\title{
NUEVA CONTRIBUCION AL CONOCIMIENTO DE LA FLORA BALEAR
}

\author{
Por LLEONARD LLORENS ${ }^{1}$
}

\section{RESULTADOS}

\section{Allium cupani Raf. subs. hirtovaginatum (Kunth) Stearn var. minoricensis var.nova.}

La subespecie era tan sólo conocida en la isla de Cabrera bajo el nombre de Allium bolosii, según cita de Palau Ferrer. La presente variedad la hallaron Bermejo y Gómez en las grietas y fisuras de los roquedos del Monte Toro en Menorca.

Las características más reseñables son las siguien tes:

Planta de 3-7 cm. de altura, de aspecto rojizo en la floración. Bulbo ovoide-cónico de hasta 8-12 mm.de largo, cubierto por túnicas pardas alargadas que se la cinian en el extremo superior en forma reticulada. Ta 110 corto, de 2-5 cm., algo carnoso, desprovisto de ho jas en el momento de la floración. Hojas verdes, fili formes, casi cilíndricas, glabrescentes aún cuando tem pranamente son algo pilosas y flácidas. Involucro for mado por una espata única, subtriangular acuminada,tan - más larga que los pedúnculos de las flores. Umbela generalmente biflora (raro uni o triflora). Flor infe rior con el pedúnculo muy corto, el de la superior un poco más largo, (4-8 mm.), ambos de color similar al del tallo . Periantio campanulado de 6-12 mm. de longitud. 
Tépalos obtusos o subagudos, blanquecinos, con el nervio dorsal patente por ser de color rojizo, en ocasiones algo violáceo. Estambres más cortos que el periantio, con las anteras amarillas y los filamentos simples, li neares o muy débilmente lanceolados. Ovario obovado $\bar{y}$ algo trígono-redondeado, de maduración rápida ya que, a veces, puede observarse su acrecentamiento cuando los tépalos están aún coloreados. Floración junio-septiem bre.

Estas plantas de Menorca presentan una diferencia clara con respecto a las demás formas de la especie co mo es la de poseer tan sólo de una a tres flores, gene ralmente son dos, en la umbela floral. Hemos cultivado plantas de Monte Toro en condiciones de mayor hume dad que las existentes en el lugar de origen pero las formas prácticamente no se han modificado y si lo han hecho algo, ha sido en el sentido de tender a poseer $\underline{u}$ nicamente una sóla flor. Otras características parecen tambiên distinguir a la forma menorquina de las demás como pueden ser una mayor carnosidad de los escapos, me nor pilosidad en las hojas en estado adulto, diferencias en la consistencia de las mismas, etc.,pero ellas no pueden considerarse todavía, en ausencia de un estu dio comparado más profundo, como significativas.

\section{Azzizm nigrum L.}

Vive en los terrenos arcillosos que se encharcan con facilidad en invierno, conjuntamente con Menta pu legium en S'Almudaina, en las proximidades de Alaior.

\section{Anthyzzis hermaniae L. var. hystrix Willk.}

Este endemismo menorquín se le conoce de diversas zonas de la costa norte y nordeste de la isla, a la vez que de rarísimas localidades del centro de la misma.En el sur (mitjorn) se ha citado en Beniparraixet ( Pons Guerau) . También vive (B. y G.) en cala En Porter for mando densos y grandes cojinetes y en los peñascos de la zona superior del Barranc d'Algendar. 


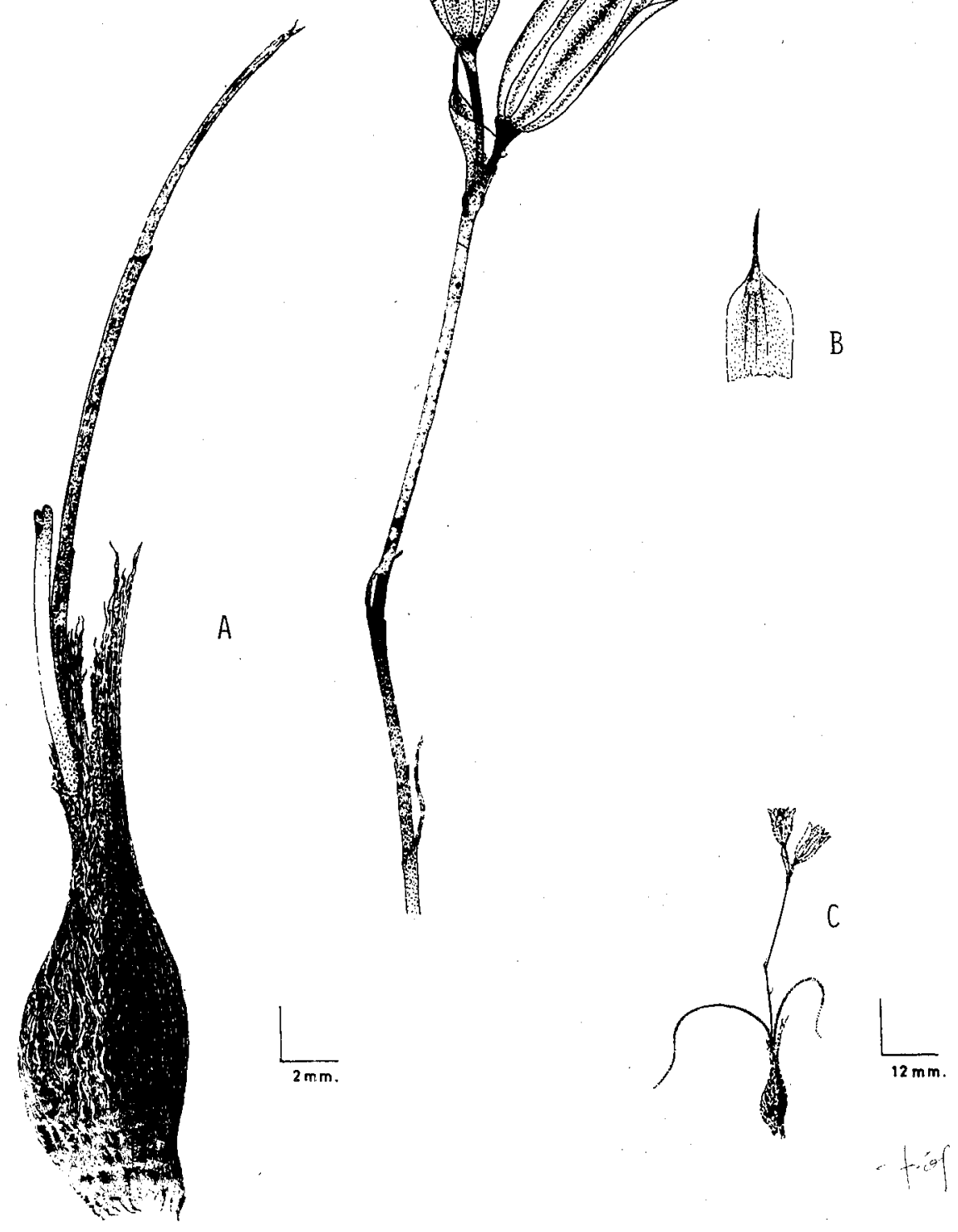

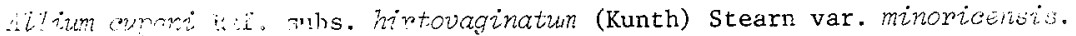

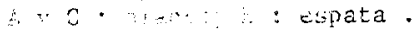


La especie,por tanto,vive de forma bastante indi ferente sobre diversos tipos de suelo, tanto arcillosopedregoso de esquistos como sobre las calcarenitas calizas con suelos de terra rosa.

\section{Anthyzlis fuzgurans Porta}

En el Cap de Cavalleria y en Macaret existen di versos ejemplares de ramas extendidas o en zig-zag muy laxo, que son iguales a las de la variedad efulgurata que Palau encontró en Cabrera. Estas formas viven a nee nudo conjuntamente con plantas muy rígidas y engrosadas de Dorycnium pentaphyllum a las que se asemejan mucho.

En Mallorca, la forma típica, además de las colo nias del Cap de Formentor ya conocidas, se la puede en contrar, como hizo Duvigneaud, en los taludes costeros rocosos situados frente a los islotes de Malgrats (San ta Ponsa) hacia el SE. de la isla.

\section{AristoZochia bianorii Sennen et Pau}

Hasta ahora la especie ha venido siendo considerada como endémica de la isla de Mallorca, sin embargo $y$ en el transcurso de unas herborizaciones de curso la hallamos en Menorca. Vive en los suelos muy rocosos, calcáreos y degradados sobre todo por la acción eólica, de Punta Nati (Ciutadella), conjuntamente con Helicodi ceros muscivorum en el límite de las comunidades de $\overline{L i}$ monietum caprariensis cuando ya penetran en ellas espè cies del Aro-Phillyretum. El lugar se asemeja por el tipo de suelo y degradación a otros de Mallorca en los que también vive la especie (Muleta de Sóller, Artá, Capdepera, Alcudia, Formentor, etc.)

\section{Aristolochia clematitis L.}

Conocida ya en Mallorca aunque sea rara.Vive tam bién en Menorca donde, gracias a las indicaciones dē Bermejo y Gómez, pudimos estudiarla. En las proximida des de Es Portixol de Faváritx, cerca de la desembocadura de un pequeño torrente que permanece húmedo duran 
te el verano, se pueden hallar distintos ejemplares,a1 gunos de los cuales alcanzan los tres metros de Iongitud, otros, más expuestos al aèrosol marítimo, no lie gan a fructificar. La floración es tardía ya que en el mes de octubre pueden hallarse todavia, plantas con sus racimitos de flores amarillentas.

\section{Aristolochia rotunda L.}

La especie es citada como rara por Rodriguez Fe menias, entre los juncos, en las Salinas de Tirant. No sotros la hemos hallado en forma de abundantes colomias en diferentes lugares del Cap de Faváritx. La más aç cesible se halla junto a la carretera que conduce al fa ro, en las proximidades de un torrente (el mismo en cü ya desembocadura vive 1a A.clematitis), el primero una vez se han superado las últimas barreras . La floración es mucho más temprana que la A.clematitis, y así, en septiembre-octubre no queda ya el menor rastro de las plantas que en primavera estaban en flor.

\section{Arthrocnemum glaucum (Dedile) Ung-Sternb.}

No citada en Formentera donde, aunque no comín, tampoco es rara en algunas zonas próximas al Estany Pü dent .

\section{Asperula arvensis L.}

Es planta rara en las Baleares. Se ha indicado en Menorca y Eivissa. Nosotros la hemos hallado en unos campos dedicados al cultivo de cereales en Consell ( Ma 1lorca).

\section{Asplenium marinum L.}

Dadas las particularidades preferencias de la es pecie, es natural que la especie sea poco conocida en estas islas. Ahora, a medida que se van abriendo nue vos accesos a los escarpados de la costa, las citas son cada vez más abundantes. Así, si hace unos años obser vamos diversos ejemplares en pequeñas cuevas de la zona baja del Caball Bernat (cala Sant Vicent, Mallorca), ahora, en un corto período de tiempo, la hemos encontra 
do cerca del Fara11ó de cala Gat (Capdepera) y en el Port d'andraig cerca del faro. Además de estos lugares ha sido Locaiizada por Font en Porto Colom, ejemplares que superan los quince centimetros. Todas estas citas hacen que, juntamente con las indicadas con anteriuridad, pueda ser considerada esparcida por toda la costa de Mallorca, a excepción probablemente de la crientada al sur.

En Menorca se produce un fenómeno semejante, pr:3 si Rodriguez la considera rara, indicándola en cala drera, Torre d'En Penjat y en la Cova de Parelleta, lo calidades todas próximas a Ciutadella, Bermejo conjuntamente con Gómez la han hallado en Algaiarens, Sa Fal conera, Cala del Pilar, Tirant, Faváritx, Cap Negre, Rá falet y Cales Coves, por lo que,al igual que en Mallor ca, puede considerarse presente en toda la costa rocosa del norte, este y oeste, asî como en los lugares más protegidos del sur.

En esta última isla (Menorca) y de modo poco usual hemos localizado algunos ejemplares en Sa Bassa Verda en Ciutadella ( $\mathrm{La}$ Vall) que se encuentra a una buena distancia de la mar y rodeada por una densa maquia. ES ta cita viene a demostrar una vez más y de modo contun dente, la fuerte influencia que el viento salino ejerce sobre toda la isla,ya que es capaz de establecer con diciones ambientales prácticamente marítimas, en luga res lejos de la costa y aún como en el caso presente, a una cierta altura y con protección vegetal.

\section{Aster tripolium L. var. minoricensis Willk.}

Se le puede hallar en las marismas de Tirant, en cuyo borde este suele abundar más en ocasiones. Sin em bargo, es en Son Bou donde es más común. La varieda $\bar{d}$ se distingue fácilmente del tipo, pues, como indican Willkomm y Rodriguez, sus lígulas son mucho más estre chas y su vilano mäs largo (en general más de dos veces que el aquenio). El caräter indicado por Rodriguez de poseer las brăcreas interiores pătulas, no es excesivamente fijo y ańs bick facresponde a un estado de ma duración que en ocasiones tambiẻn se da en el tipo. 
De las Baleares, tan sólo faltaba citarla en Formen tera. La hallamos en unos campos cultivados cerca de San Francisco, y también en unos pequeños cercados que se usan como campos de cultivo, en los arenales de los alrededores de las salinas de Marroig . La adaptación a estos ambientes es buena y, probablemente por ello, se ha intentado usar como forrajera . En Mallorca, en épo cas de escasez de café, sus semillas, tostadas, se usaban como pobre sucedáneo del mismo.

\section{Atriplex halimus L.}

Es planta rara en Formentera, en donde todavía no se había indicado. Se la puede hallar en los alrededo res de las Salinas de Marroig en dirección a Trucadors.

\section{Bupleurum rigidum L.}

Kuhbier (1978) la indica en cala Eubarca, en el bos que de Pinus halepensis. Siendo su área de colonización muy reducida, no es extraño que pasara desapercibi da a los botánicos que, con cierta frecuencia, han visitado la zona. Nosotros la hallamos en unos bancales a bandonados situados bajo los peñascos acantilados.

Eivissa es la única localidad conocida de las Baleares.

Carex divisa Huds. var. chaetophyzza (Steud) Daveau.

Vive en los terrenos salobres húmedos de las sali nas de Eivissa, lindando con los terrenos del aeropuer to.

La variedad es nueva para la isla, en donde ya se ha citado el tipo.

\section{Centaurea balearica Rodriguez}

Las citas de la planta en Menorca son escasas(las de Mallorca pueden considerarse,casi con seguridad, co mo una confusión) . Rodriguez dice que la halló en el 
Pou d'En Carles y en el Port de Fornells, y Porta entre el Cap de Cavalleria y la Penya del Anticrist. La pri mera de las tres citas es la más clásica y conocida,pe ro las otras dos están peor determinadas y desde luego no concretadas. Bermejo y Gómez, en un recorrido exha ustivo por la zona, obtuvieron la confirmación (ya su puesta) de la existencia de numerosas colonias, algunas de ellas formadas por abundante cantidad de ejemplares. Tanto en la zona indicada por Porta, en particular en tre Ferragut y la cala d'En Calderer, como fuera de ella, cala Tirant, la especie tiene una vitalidad semejante a la de otros cojinetes espinosos endémicos de las Balea res.

Vive muy bien en los arenales (Ferragut) pero su óptimo parece hallarse más en los suelos porosos y ro cosos próximos al mar y muy expuestos a los duros vientos de Tramontana. Existe una variabilidad patente en 1o que se refiere a la densidad de los cojinetes y a la longitud de las espinas de las brácteas del involucro floral que parece estar relacionada directamente con la exposición y con la naturaleza del suelo (suelos más po bres y secos, espinas más cortas y cojinetes más densos).

\section{Cuscuta compestris Yuncker}

Nueva para las Baleares en donde es probablemente de reciente introducción. Vive en las dunas de Son Se rra de Marina (Mallorca) parasitando diversas plantas de la Ammophiletea (Calystegia soldaneila, Eryngium ma ritimum y Lotus cytisoides). Se destaca claramente so bre la arena durante el otoño y el invierno, debido a su color amarillento. Es muy probable que los caballos sean el vehículo principal de diseminación de la espe cie.

Cuscuta epithymum L. subs. planiflora(Tenore) Rouy

Rara en Mallorca y Cabrera . La hallamos escasa en los alrededores del caserío de Sa Calobra, junto a unos peñascos, parasitando dos plantas endémicas:Rubia baleárica y Scutellaria baleárica 
Vive en Menorca, en donde no se conocía, en las grietas de los peñascos de los montículos de Llináritx. Los ejemplares alcanzan magníficos tamaños, superando a1 gunos los treinta centimetros de longitud.

En Mallorca, además de en Sóller, única localidad en donde se conocía, se le puede hallar en el Estret de Valldemossa (unas centenas de ejemplares) y en unos pe ñascos situados en la zona superior de Es Plá des Vicá ri en Andratx.

\section{Daphne rodriguezii Teixidor}

Es considerada como planta rara en Menorca. Las citas que de ella se han hecho, se reducen a tres localidades: la isla d'En Colom, cala de Sa Mesquida y Mon gofre Nou . Ahora, a ellas hay que añadirles otras cua tro: Macaret, Daia, La Mola de Fornells y cala En Por ter.

En Macaret hemos localizado más de treinta ejemplares que suelen confundirse por entre el matorral alif existente y que se compone eminentemente por Pistacia lentiscus, Erica multiflora, Rosmarinus officinalis Phi IIyrea media var. rodriguezii, Cistus monspeliensis, Cistus incanus, Calicotome spinosa, Dorycnium pentaphy ZZum y Thymelaea velutina. La altura media de la vege tación está entre un metró y un metro cincuenta centímetros, y el sustrato es arenoso o arcillo-arenoso.

En la Mola de Fornells el sustrato y el matorral son semejantes, aunque este último sea un poco más ele vado y lleve también cantidades perceptibles de Erică scoparia. La presencia de Daphne es también mayor y se pueden encontrar zonas de densidad relativamente eleva da(hemos contabilizado algunas áreas de unos $1000 \mathrm{~m}^{2}$ con más de quinientos ejemplares en cada una de ellas). Es ta cita es sin duda la que mayor riqueza de plantas pre senta.

Sin embargo, es en cala En Porter donde resulta más interesante desde el punto de vista geográfico y $\underline{e}$ cológico, pues el lugar, además de estar aislado de los 
anteriores (en el sureste, "mitjorn", de la isla)tiene un suelo formado por terra rosa, instalada sobre una ro ca madre calcarenítica, de fuerte reacción carbonatada diferente del de las demás localidades, que siempre lle va cantidades más o menos notables de micas. En esta cá la crece siempre al abrigo de otras matas, generalmente de Pistacia lentiscus y Juniperus phoenicea, y jun to a ejemplares muy desarrollados y viejos, se encuentran otros muy jóvenes más cobijados todavía.

Con estas nuevas citas, la especie que se consi deraba casi extinguida de la isla de Menorca (de hecho se suponía casi reducida al islote de En Colom) aumenta considerablemente su área de distribución conocida.

\section{Diplotaxis catholica (L.) DC.}

Es especie común en algunas áreas de Mallorca (Campos) y de Eivissa (playas de Es Cavallet y Ses Sa lines), casi siempre en los arenales o en lugares coste ros arenosos.

No era conocida hasta ahora en Formentera en don de, si bien más o menos rara, la encontramos en las plä yas de Es Pujols y Mitjorn por entre el matorral forma do sobre todo por Juniperus phoenicea. La cita no es rara ya que estas localidades, especialmente la de Es Pu jols, se encuentran muy próximas a las antes indicadas de Eivissa.

\section{Echinophora spinosa L.}

Es especie rara en las Baleares. Se conocía so lamente de Eivissa y Mallorca . En esta última isla, la localidad típica de la playa de S'Arenal, ha sido des truida por las construcciones de los complejos turisti cos, por lo que tan sólo conocemos dos localidades más situadas en las dunas de Sa Rápita de Campos y Son Se rra de Marina, en la bahía de Alcudia .

También es escasa en Eivissa, estando localiza da en las playas y calas que miran a Formentera, algunas aún poco urbanizadas.

En Formentera tan sólo la conocemos en forma de 
magnificos ejemplares, en el arenal de cala Saona, y en Menorca, en las dunas de Son Bou (muy rara). Para estas dos ultimas islas es cita nueva.

\section{Euphorbia maresii Knoche}

En Menorca se cita la variedad minoricensis que es considerada como planta rara. Nosotros la hemos ha llado en forma más o menos contínua, por toda la zona costera entre cala Morell y Cap Favaritx. Busca la pro tección en los cojinetes espinosos, por 1o que no es ex traño que haya pasado muy desapercibida. Sin embargo, en algunos lugares como en cala Morell, se halla comple tamente al descubierto, en donde vive en las grietas ré llenas de arcillas, situadas sobre las cuevas allí exis tentes.

En Mallorca, la variedad baleárica se cita en la Serra de Tramontana y la variedad maresii (típica)en Artá, cerca de 1a ermita y en el Cap de Es Pinar (Alcu dia). También se puede encontrar esta última variedad en el Cap de Formentor, por entre Anthylzis fulgurans y Teucrium subspinosum, y en las cercanías de Sa Font de Sa Cala (Capdepera). En el sur de la isla, en las grietas de los roquedos del Cap Blanc y Cap de Regana, se encuentra una forma intermedia entre las dos variedades de la isla de Mallorca y que es a su vez muy próxima a la variedad minoricensis. Ello parece indi car que un estudio profundo y comparado de las distintas variedades, está todavía por realizar. Dado que 1a especie es muy polimorfa, debería relacionarse también con la E.gayi, de la cual fue considerada por algunos au tores, parte integrante.

\section{Euphorbia prostata Ait.}

Es de reciente introducción en Mallorca . Unica mente la conocemos en cala Murada, no lejos de la playa cerca de una acera, mezclada con la E.chamaecyse.

\section{Eerula comminis L. subs. comminis Cannon}

Conocida en Eivissa y Menorca, no estaba indica da en Formentera, en donde la hallamos cerca del Esta- 
ny Pudent, en un suelo rocoso muy pobre.

Galium vermucosum Hud.

Abunda en los campos abandonados de Formentera (San Francisco, El Pilar, Es Pujols, etc.) en donde no se había citado.

Helianthemum caput-felis Boiss.

La única colonia conocida hasta ahora en las Baleares, es la que halló Marés en Campos. Nosotros la he mos encontrado en varias localidades de la costa entre Cap Enderrocat y Vallgornera. Abunda en diversos pun tos de los taludes marítimos arenosos, cuando bajo está arena hay tierras arcillo-pedregosas o rocas agrietadas, especialmente entre el Cap de Regana y el Cap Blanc, Es más raro en otras condiciones o localidades, como suce de en la playa de Es Trenc, donde puede hallársele sobre los afloramientos rocosos que en ocasiones existen en la parte posterior de las primeras dunas.

A veces, los ejemplares de los taludes alcanzan tamaños considerables, mayores incluso que los observa dos por nosotros en el Portet de Moraira en Alicante, pues pueden superar $1,5 \mathrm{~m}$. de diámetro. A menudo, es tas plantas tienen las hojas sólo un poco dobladas por los bordes o incluso totalmente planas, con lo que su aspecto se hace bastante diferente del típico.

Hyazinthais pouzolzii Gay

En Menorca ya era conocida de S'Enclusa y de las Salines de Fornells. Vive también en las grietas delos peñascos, juntamente con Digitalis dubia, en Llináritx Nou (Mercada1).

En Mallorca la cita Duvigneaud (1979). El nos la indicó en unas grietas, casi totalmente ocupadas por $\mathrm{Ca}$ rex romilenta, del Penyal Fumat en el Cap de Formentor.

Kosteletzkya pentacarpos (L.). Lebed.

En 1976 hallamos un ejemplar en la Phragmitetea 
de Algaiarens (Menorca). Con posterioridad hemos regre sado al lugar y constatado que continúa reproduciéndose,lo que nos induce a pensar que pueda proliferar $y$ transformarse en una nueva inquilina de la flora de Me norca. La estación es semejante a las conocidas de Va lencia y delta del Ebro. 1au).

De las Baleares, se había citado en Cabrera (PaLavatera trizoba L. subs. pallescens (Morris) Nyman

Rodriguez indica esta especie en la costa este de Menorca (hasta Binidalí por el sur), y en especial en la zona entre Sa Mesquida y el Cap Negre. No es rara tampoco al norte de Sa Mesquida, hasta las colinas del Cap Faváritx. También la hemos encontrado en los alre dedores del faro de Punta Nati, sobre suelo muy rocoso carstificado . Esta localidad, al noroeste de la isla, es completamente diferente de las anteriormente indica das.

Limonium delicatulum (Girard) 0.Kuntze subs. toumefortii Pignatti.

Como sucede con diferentes especies del género, las citas en las Baleares son confusas. Se ha indicado, además de en Mallorca y Formentera, en Menorca,pro bablemente confundida con,el L.biflomm .

Vive también en Eivissa, común en los saladares de Ses Salines, donde muy probablemente también se ha confundido, aunque esta vez con formas del L.romosissi mum .

Hay que considerar pues a esta especie, del Medi terráneo sur-occidental, distribuida por lo menos por Mallorca, Eivissa y Formentera, preferentemente en sue los ricos en sulfatos.

\section{Limonium ebusitanum F.Q.}

Pertenece al grupo de especies poco conocidas de las Baleares, aunque es una de las que tiene la clave 
del género en ellas.

Es común en todo el sur de la isla de Mallorca, donde se ha citado con poca fiabilidad y exactitud,asi como en toda la isla de Eivissa. También abunda en For mentera en la costa rocosa y en el sur de Menorca ( cá la En Porter, Binissafuller, cala de Santa Galdana, Cap d'Artrutx, etc.), alcanzando también el noroeste en Pun ta Nati, en cuyo extremo viven ejemplares muy bien desarrollados.

El Limonium sp. que Rodriguez indica en el Caló de Sant Esteve, debe también incluirse en esta especie.

\section{Lycium intricatum Boiss.}

En Mallorca se encuentra en los taludes costeros entre cala Blava y Vallgornera, y más rara en los roque dos de la parte alta de los mismos (en ocasiones se le puede ver por entre las piedras de los muros).

En Formentera también vive en condiciones simila res; allí aunque no común, tampoco es raro en los peque ños taludes formados por desprendimientos de las rocas en la costa sur de La Mola . En esta localidad puede al canzar la parte norte, pero más aisladamente.

Los ejemplares que en las dos islas viven en las grietas de las rocas, son generalmente de pequeño tama ño y tienen aspecto esquelético.

\section{Malcomia ramosissima (Desf.) Thell.}

La hallamos en los claros de las dunas de Es Pujols (Formentera) en particular en el Juniperetum $l_{y}$ ciae. Se conocía ya en Menorca y Eivissa.

\section{Maresia nana (DC.) Batt.}

Conocida en Mallorca y Eivissa. Vive también en Formentera, conjuntamente con Malcomia romosissima, y parece preferir en la localidad (Es Pujols), lugares más protegidos que ésta. 
Melissa officinalis L.

La encontramos en Son Blanc Nou (Alaior) en terre nos de encinar bastante degradado.

No se había indicado en Menorca.

\section{Merendera filifolia Camb.}

Abunda en los 1lanos pedregosos y rocosos desforestados de Formentera (La Mola, Cap de Berberia, San Francisco, etc.) . Conjuntamente con Narcissus serotinus y Arisamum vulgare, componen la floración geofítica autumnal en la isla.

\section{Neottidea intacta (Link) Reichenb.}

No es rara en los bosques y matorrales densos de la costa norte y noroeste de Eivissa. Es con Ophrys fusca subs. omegaifera y Ophrys bertolonii, el grupo de orquídeas más abundantes en la zona.

\section{Oenanthe Zachenalii C.C.Pome1}

No es muy rara en las marismas de Son Bou, cerca ya de Ses Canasies (Menorca) . Rodriguez cita en esta localidad, una especie que no pudo clasificar y que po dría ser la presente, pero sus indicaciones no son muy determinantes, $y$ no es posible clarificarlo con seguridad .

\section{Onopordon macracanthum (Boiss.) Schousb.}

Especie ibero-mauritănica que alcanza las Baleares en Mallorca. Vive en la Marina de Llucmajor en par ticular en los alrededores de las fincas que poseen ga nado (Son Albertí, Capocorb, etc.), y más raro en otras localidades como Magalluf y Xorrigo :

Es posible que las inconcretas citas que se han hecho del 0.acanthium, o por 10 menos parte de ellas, correspondan a la presente especie. Es fácil de distin guir, dado el diámetro de sus capítulos (de hasta $6 \mathrm{~cm}$.) y la disposición refleja de sus brácteas exteriores. 
La especie, como ha sucedido tambiên en gran par te del Mediterráneo occidental, ha sido diezmada por caū sa de una recolección incontrolada, debido a sus virtū des medicinales. En Mallorca está prácticamente extiñ guida, tan sólo conocemos dos ejemplares en la Playa de Es Trenc (Campos). En las Pitiusas es también escasísima, pues tan sólo se conoce en forma silvestre en las playas de Es Codolar (Eivissa) y Trucadors (Formentera). En esta ultima isla es mu corrientemente cultivada en los jardines (herba de Trucadors) pues es considerada casi como una panacea medicinal.

\section{Pallenis spinosa (L.) Cass.}

En Menorca conviven dos formas: una típica, de Iigulas anaranjadas, y otra que las posee amarilias y más estrechas. Bolós y Montserrat (comunicación ver bal) ya notaron su existencia. La de lígulas amarillas, aun sin estar ausente de los terrenos muy calizos, parece ser más abundante e incluso predominante en los me nos carbonatados.

$\frac{\text { Pinus halepensis Miller var. ceciliae comb. noval }=P .}{\text { ceciliae A i LL. Llorens) }}$

Después de su hallazgo en la localidad tipo de Son Alberti (Mallorca), cuyo tipo conservamos en nuestro herbario, su área de dispersión conocida ha aumentado considerablemente. Asi lo hemos visto en la finca de Ets olors (Artá) cerca de una colina denominada como Puig des Pins Campanars, nombre que si bien puede ser una coincidencia, sugiere muy bien la forma de la espe cie . La indicación de algunos viejos leñadores, en ễ sentido de que conocían este tipo de pino y de que era preferido por ellos para talarlo (su madera es más blan da), juntamente con la localización en Artá y en otros lugares de la Marina de Llucmajor, nos hace suponer que antaño, tal clase de pino era más abundante.

Esta suposición se nos confirmó más si cabe, cuan do 10 encontramos en Eivissa, cerca de Es Caná, en un denso pinar y sobre todo, en el momento en que orfila (aficionado naturalista menorquín) nos 1o indicó en Me 
norca - En efecto, en un pinar situado en las laderas calcáreas inmediatas al mar, en el tramo comprendido entre el barranco de Sa Vall y Santo Tomás, se distin guen numerosos ejemplares muy bien desarrollados.

Con estas nuevas citas, e1 origen del táxon pare ce ahora más confuso, pues si antes cabía la posibilidad de una mutación local y aislada, las nuevas localizacio nes obligan a soluciones más complejas .

\section{Plantago albicans L.}

Excluida por Rodriguez en su flora, y desde enton ces considerada dudosa su existencia en Menorca, debe aseverarse su presencia. En Atalis, en terrenos areno so-arcillosos muy pobres, de degradación de otros algo más ricos, poblados por trazas de la comunidad de Erica multiflora y Lotus tetraphyllus (Rosmarino-Ericion), se puede encontrar dicha especie juntamente con Convolvulus althaeoides y Scabiosa maritima, formando también muestras parciales de la comunidad de Plantaginetum al bicantis (Thero-Brachypodietea).

\section{Plantago crassifolia Forsk.}

Es planta rara en Formentera, en donde no se ha citado hasta ahora. Vive en las proximidades de S'Estany Pudent, en suelos arenosos. E1 Schoenetum-Planta ginetum crassifoliae está, en dicha isla escasamente re presentado, menos aún que en su vecina Eivissa.

\section{Polycarpon alsinifolium (Biv.) DC.}

Conocida ya en Mallorca, Eivissa, Dragonera y For mentera, la hemos encontrado en Menorca. En las dunas de Son Bou no es rara, sobre todo en la parte posterior de la primera duna, al inicio del matorral de Jimiperus.

\section{Psoralea bituminosa L.}

En Menorca hallamos, en el camino que conduce a Binigarba desde 1a carretera de Maó a Citadella,varios ejemplares de un mutante albino perfectamente desarro11 ado. 
En las Pitiusas, las encinas son escasas, y en Formentera no se habían citado. Conocemos dos ejempla res, cultivados, bien desarrollados. Uno de ellos es fácilmente observable, ya que se encuentra tras una pa red en la margen sur de la carretera de La Savina a La Mola, en las proximidades del caserío de San Fernan do.

\section{Rhomnus Zudovici-salvatoris Chodat}

La única cita que existe en Menorca se debe a Ro driguez, quien indica haber hallado un solo ejemplar mas culino en el arenal de Tirant. Recorrida la zona por nosotros, hemos podido encontrar varios ejemplares (unos quince) típicos, prácticamente iguales a los de Mallorca, junto a otros que parecen formas intermedias con el R.aZaternus. Será preciso estudiarlas en épocas de floración y fructificación, para poder determinar su posición taxonómica.

La localidad, sin embargo, corre un gran riesgo de ser destruida por las construcciones que se realizan y por las extracciones de arena que se Ilevan a cabo en las proximidades.

\section{Salicomia ramosissima J. Woods}

Rodriguez, en su Flórula de Menorca excluye esta especie de la isla, a pesar de haber sido indicada por Cursach como S. herbacea. Duvigneaud, sin indicación de lugar, cita la S.ramosissima en Menorca. Nosotros la encontramos en Sa Mesquida, en los lugares salinos encharcados durante gran parte del año, a pocos metros de las comunidades de Isoetion.

\section{Salsola oppositifolia Desf.}

Vive en Formentera, en los acantilados del Cap de la Mola, así como en algunos muros de los cercados próxi mos. Juntamente con Lycium intricatum forman el matorral leñoso más elevado de los alrededores del faro. La especie se había citado tan sólo en Eivissa. 
Es planta rara en las Baleares . En Mallorca, ú lica isla en donde se conocía, aparece de vez en cuanlo, cada vez menos, de forma explosiva en algunas zonas le la Albufera de Alcudia.

También vive en Menorca, junto a unos canales, en 21 saladar situado tras de las dunas de Es Grao de Maó.

\section{Spergularia marina (L.) Griseb.}

Rara y nueva para Formentera. Se desarrolla muy bien, formando magnificos ejemplares junto a los estan ques de las Salinas de Marroig.

\section{Trifolium scabrum L.}

No es rara en Formentera . Abunda en los alrede dores de S'Estany Pudent y en los campos cultivados de las cercanías de San Francisco.

\section{Trifolium squarrosum L.}

Crece en las orillas de la carretera que conduce al Cap Faváritx a la altura de Capifort y Maongofre Vell, en cuyas fincas probablemente ha sido ensayado co mo forrajera.

\section{Vicia bifoliolata Rodr.}

Esta especie ha venido siendo considerada casi como desaparecida de la flora de Menorca, hasta que B. Mateo la redescubrió enredada en los Cistus monspelien sis de Binisarmenya.

Nosotros la hemos encontrado, encaramada a los jun cos que bordean el Isoetion marítimo de la zona, y más tarde, en distintos lugares de la comarca, siempre en ambientes marítimos. Primero hasta las proximidades de cala Murta y más tarde, hasta cerca de Ses Bancades, en tre el Cap Negre y el Cap de La Mola.

Es planta que presenta dificultades para ser 1으 
calizada, si no se está habituado a su fino aspecto, puesto que 1o intrincado del matorral en donde vive $y$ 1a pequeñez de sus flores y hojas, a la vez que su semejanza con la V.tetrasperma de la que tan sólo se dife rencia casi por el número de foliolos, la hacen dificilmente observable. Sin duda, es planta rara,pero no tanto como hasta ahora ha venido siendo considerada.

\section{Viola arborescens L.}

No es rara en Formentera, tanto en el interior del bosque de pinos como en los lugares más claros.Las mayores y densas colonias que hemos observado, se encuentran junto a la carretera que conduce de San Fran cisco a La Mola antes de llegar a Es Caló; allí, en u nos bajos de un corte a modo de talud, el suelo en e $\overline{1}$ mes de noviembre parece salpicado de flores violetas.

\section{Withania frutescens (L.) Pauguy}

En Mallorca sólo está indicada por Cañigueral en e1 Puig Morisca (Santa Ponsa). Existe también, aunque muy rara, en las proximidades del Cap Enderrocat (entre este y el Cap de Regana), en unos taludes costeros. La cita se sitúa en una posición geográfica intermedia en tre la anterior indicada de Santa Ponsa y la de Cabrera, en donde también se ha señalado.

\section{Xanthium stmuarium L.}

En Eivissa lo conocemos en diversas localidades (Sant Antoni, Santa Eulalia, Eivissa, cala Portinaitx, etc.), asi como en Formentera (La Savina, Es Pujols,Es Caló, cala Saona, etc.), siempre relacionado con luga res con mayor o menor influencia humana. Es probable que sea de reciente introducción, ya que no había sido indicada en las Pitiusas. 


\section{RESUMEN}

Comprende esta comunicación informaciones sobre di versos táxones presentes en las islas Baleares.

Se incluyen formas nuevas, como el AlZium cupani subs. hirtovaginatum var. minoricensis, combinaciones nuevas, como Pinus halepensis var. ceciliae $(=P$. ceci liae), citas de diversas especies desconocidas hasta ahora en las islas, así como distintas nuevas localida des para plantas ya indicadas en ellas.

\section{SUMMARY}

This article is composed of information regarding various taxons found in the Balearic Islands.

New forms such the AlZium cupani subs. hirtovaginatum var. minoricensis are included, also such new com binations as Pinus halepensis var. ceciliae ( $=$ P.ceciliae), also cited in varied species unknown until now in the islands, as well as different new locations for plants already indicated in these islands.

\section{AGRADECIMIENTOS}

Debemos agradecer al señor Lluis Fiol la confección del dibujo que se adjunta, y a los señores Rosse11ó, Socies, Font y muy especialmente a los señores Llo rens de Ros, Bermejo y Gómez, las diferentes indicaciones que han puesto a nuestra disposición. 


\section{B I BL I OGRAF I A}

BOLOS,O.de, MOLINIER,R. y MONTSERRAT,P., 1970.- Observations phy tosociologiques dans $1^{\prime}$ ile de Minorque.-Act. Geobot.Barcinonen sia. vol.5. Barcelona.

CARRETERO,J.L., 1979. - So Zanum elaeagnifolium Cav. y Cuscuta cam pestris Yuncker, nuevas especias para la flora española. Coli. Bot. vol.XI n ${ }^{\circ}$ :143-157. Barcelona.

DUVIGNEAUD, J., 1979.- Catalogue provisoire de la flore des Baléares.-Soc.Ech.PZ.Vas. Eu.Occ. et B.Méd. Fasc.17 supp1. Liè ge .

FONT i QUER, P., 1925.- De Florae Occidentalis adnotaciones. Ca vanizlesia I, $\mathrm{n}^{\circ} 10$. Barcelona.

FONT $i$ QUER, P., 1926.- IlZustrationes Florae Occidentalis. p. 5 Tab. 1. Barcelona.

KUHBIER, H., 1978.- Beitrage zur Flora der Pityusen. Veroff.Uber seemuseum Bremen.- Reihe A Band 5: 1-23. Bremen .

LLORENS, A. y L1., 1972.- Contribución al conocimiento de la flo ra balear. Bot.Soc.Hist.Nat.Bal. Tom.XVII p:51-54. Palma de Mallorca.

LLORENS, L1., 1979.- Los Limonium de Za isla de MaZZorca.- Tesis doctoral (inéd.) Facultad de Farmacia. Barcelona.

MARCOS, A., 1936.- Contribució al coneixement de la flora balear. Flórula de Cabrera y dels illotspròxims. Cavanizzesia VII, p. 5-52. Barcelona .

MATEO, B., 1979.- Presencia de Vicia bifoliolata Rodr.Fem. en Me norca. Colz.Bot. vol.XI n¹0:269-270. Barcelona.

PAlAU, P., 1976.- Flómula de Cabrera. Inst.Catal.Hist.Nat.- Bar celona .

PIGNATTI, S., 1955.- I Limonium della flora balearica.- Arch. Bot. XXXI, p.65-100 . Forli.

QUEZEL, P. y SANTA, S., 1963.- NouvelZe Flore de Z'AZgerie et des régions desértiques méridionales. Paris.

RODRIGUEZ FEMENIAS, 1904.- Flómula de Menorca . Mahón .

TARAZONA LAFARGA, M.T., 1975.- Herborizaciones en la isla de For mentera (Baleares). Trab. Depart. Bot. 7: 23-33. Madrid.

TUTIN et al., 1964, 1968, 1972, 1976.- Elora Europaea. vols. 1 a 4. Cambridge. 